\title{
Extensivity of two-dimensional turbulence
}

Article

Accepted Version

Tran, C. V., Shepherd, T. G. and Cho, H.-R. (2004) Extensivity of two-dimensional turbulence. Physica D: Nonlinear Phenomena, 192 (3-4). pp. 187-195. ISSN 0167-2789 doi: https://doi.org/10.1016/j.physd.2004.01.002 Available at https://centaur.reading.ac.uk/32115/

It is advisable to refer to the publisher's version if you intend to cite from the work. See Guidance on citing.

Published version at: http://dx.doi.org/10.1016/j.physd.2004.01.002

To link to this article DOI: http://dx.doi.org/10.1016/j.physd.2004.01.002

Publisher: Elsevier

All outputs in CentAUR are protected by Intellectual Property Rights law, including copyright law. Copyright and IPR is retained by the creators or other copyright holders. Terms and conditions for use of this material are defined in the End User Agreement.

\section{www.reading.ac.uk/centaur}

\section{CentAUR}

Central Archive at the University of Reading

Reading's research outputs online 


\title{
Extensivity of two-dimensional turbulence
}

\author{
Chuong V. Tran ${ }^{1}$, Theodore G. Shepherd, Han-Ru Cho \\ Department of Physics, University of Toronto, 60 St. George Street, Toronto, ON, \\ Canada, M5S $1 A 7$
}

\begin{abstract}
This study is concerned with how the attractor dimension of the two-dimensional Navier-Stokes equations depends on characteristic length scales, including the system integral length scale, the forcing length scale, and the dissipation length scale. Upper bounds on the attractor dimension derived by Constantin-Foias-Temam are analysed. It is shown that the optimal attractor-dimension estimate grows linearly with the domain area (suggestive of extensive chaos), for a sufficiently large domain, if the kinematic viscosity and the amplitude and length scale of the forcing are held fixed. For sufficiently small domain area, a slightly "super-extensive" estimate becomes optimal. In the extensive regime, the attractor-dimension estimate is given by the ratio of the domain area to the square of the dissipation length scale defined, on physical grounds, in terms of the average rate of shear. This dissipation length scale (which is not necessarily the scale at which the energy or enstrophy dissipation takes place) can be identified with the dimension correlation length scale, the square of which is interpreted, according to the concept of extensive chaos, as the area of a subsystem with one degree of freedom. Furthermore, these length scales can be identified with a "minimum length scale" of the flow, which is rigorously deduced from the concept of determining nodes.
\end{abstract}

Key words:

Global attractor, Navier-Stokes equations, Extensive chaos, Characteristic lengths PACS: 47.52.+j, 05.45.Jn, 47.17.+e, 47.27.Gs

\footnotetext{
Email addresses: chuong@math.ualberta.ca (Chuong V. Tran), tgs@atmosp. physics . utoronto.ca (Theodore G. Shepherd), cho@rainbow. atm.ncu.edu.tw (Han-Ru Cho).

1 Present affiliation: Department of Mathematical and Statistical Sciences, University of Alberta, Edmonton, Alberta, Canada, T6G 2G1
} 


\section{Introduction}

It has become a classical idea that the attractor dimension - a measure of dynamical complexity - of a certain class of dissipative physical systems scales linearly with the volume of the system, a property known as "extensive chaos" $[8,15,16]$. This central idea of extensive chaos is physically interpreted and best appreciated with the following heuristic argument. In a dissipative system there exists a dissipation length scale $\ell$, below which all modes are damped; only modes with length scales above the dissipation scale are dynamically active. Intuitively, the attractor dimension is approximately equal to the number of active degrees of freedom. If other parameters can somehow be controlled so that $\ell$ remains fixed while the volume $V$ of the system of typical length scale $L$ is increased, then the number of active modes grows as $(L / \ell)^{d}$ where $d$ is the spatial dimension of the system-i.e. the degrees of freedom grow linearly with $V$. For demonstrative purposes let us consider a dissipative dynamical system defined in a (Hilbert) space $H(\Omega)$ of doublyperiodic functions on $\Omega=(0,2 \pi L) \times(0,2 \pi L)$. This space is spanned by $\left\{\cos L^{-1}\left(k_{1} x_{1}+k_{2} x_{2}\right), \sin L^{-1}\left(k_{1} x_{1}+k_{2} x_{2}\right)\right\}$ where $k_{1}$ and $k_{2}$ are integers. A basic mode of wavevector $\left(k_{1}, k_{2}\right)^{T}$ above the length scale $\ell$ satisfies

$$
\left(k_{1}^{2}+k_{2}^{2}\right)^{1 / 2} \leq L / \ell
$$

Modes satisfying the above inequality can be geometrically identified as lattice sites inside the circle of radius $L / \ell$, the number of which is approximately the area of the circle (for a sufficiently large radius). It follows that the number of modes above the length scale $\ell$ is proportional to $(L / \ell)^{2}$ which is proportional to the domain area. Hence, extensive behaviour might be expected to be common among dissipative dynamical systems for which the dissipation length scale is independent of the system size. (Thus, the problem of proving that a system is extensively chaotic might be restricted to showing that its dissipation length scale is size-independent.) Although expected to be common, extensive behaviour has only been partly validated for a few cases. On the theoretical side, for example, extensive behaviour has been suggested for the 2D Navier-Stokes equations on an elongated domain for a special class of the driving force [38] and for the one-dimensional complex Ginzburg-Landau equation [1]. ${ }^{2}$ On the experimental side, it has been shown numerically that the Kuramoto-Sivashinsky model [24] and the Miller-Huse model [26] behave

$\overline{2}$ In those works, an upper bound on the attractor dimension that scales linearly with the domain "volume" is derived. Strictly speaking, such an upper bound does not fully establish extensivity, without a lower bound that behaves in the same manner. For the 2D Navier-Stokes equations on an elongated (square) doubly periodic domain driven by a simple forcing, a lower bound on the global attractor that scales linearly with the domain area is derived by Babin and Vishik [2] (Liu [23]). 
extensively.

Another physical interpretation of the idea of extensive chaos, according to its originators, is that large systems become complex with increasing size in a simple way by replicating weakly-interacting and statistically similar subsystems of some characteristic size $[8,15,16]$. In other words, an extensively chaotic system is a system such that there are no interesting collective effects with growing size, as might be expected if small dynamical units somehow bind themselves into a larger effective unit with fewer total degrees of freedom. The characteristic size is identified as the dimension correlation length $[8-10,15,16]$, defined as $\xi_{\delta} \equiv(V / D)^{1 / d}$, where $V$ is the volume of the system, $D$ is the (global) attractor dimension, and $d$ is the dimension of the system's physical space. Hence, a subsystem with volume $\xi_{\delta}^{d}=V / D$ is interpreted as a dynamical unit with one degree of freedom. Clearly, if $\xi_{\delta}$ remains fixed as $V$ is varied, then $D \propto V$ and the system is extensive. So in this case the problem of proving extensivity amounts to showing that $\xi_{\delta}$ is size-independent.

In this study we examine the extensivity of two-dimensional turbulence. The two standard estimates of attractor dimension derived by Constantin-FoiasTemam [6,7] for the Navier-Stokes equations with doubly periodic boundary condition are analysed. It is shown that one of the estimates, when modified to take into account the forcing length scale, is optimal, from the perspective of extensive chaos. Moreover, the modification makes the physical significance of the forcing length scale more apparent. In particular, a forcing with fixed amplitude, when applied at progressively smaller length scales, injects a smaller amount of energy, leading to an increase in the dissipation length scale (decrease of Reynolds number and of attractor dimension). The other estimate, which is slightly super-extensive, turns out to be optimal when the system's length scale is sufficiently small. We also address the physical relevance of the dimension correlation length, and its interpretation as the size of relatively independent subsystems in an extensively chaotic mother system. In fact, the dimension correlation length can be identified with the dissipation length as defined, on physical grounds, by Hohenberg and Shraiman [16]. Furthermore, this length scale is equivalent to a "minimum length scale" rigorously deduced from the concept of determining nodes $[13,14]$.

\section{Attractor-dimension estimates for 2D turbulence}

The asymptotic behaviour of the solutions of the forced two-dimensional Navier-

Stokes equations:

$$
\frac{\partial u}{\partial t}+(u \cdot \nabla) u+\nabla p=\nu \Delta u+f(x), \quad \nabla \cdot u=0, \quad x \in \Omega \subset \mathcal{R}^{2},
$$


has been the subject of intense research for the past 30 years $[2,5-7,11,12,21,33,35,36,38]$. The existence of a bounded finite-dimensional (Hausdorff or fractal dimension) global attractor has been established $[12,21]$, and estimates on its dimension calculated $[2,6,7]$. The idea behind this calculation is that if an arbitrary $m$ dimensional volume element of the phase space asymptotically compresses to zero, then the number of positive Lyapunov exponents of the system and the Hausdorff (as well as the fractal) dimension of the global attractor cannot exceed $m$. The greatest lower bound on all such possible $m$ is then an upper bound on the attractor dimension. ${ }^{3}$ The book of Temam [32] provides details of this mathematical technique and its application to various dissipative dynamical systems. Two standard attractor-dimension estimates are obtained when doubly periodic solutions on $\Omega=(0,2 \pi L) \times(0,2 \pi L)$ of $(1)$ are considered:

$$
\begin{aligned}
& D \leq c_{1} G, \\
& D \leq c_{2} G^{2 / 3}(1+\ln G)^{1 / 3},
\end{aligned}
$$

where $D$ is the attractor dimension, $c_{1}$ and $c_{2}$ are absolute constants, and $G$ is the generalized Grasshof number defined by

$$
G \equiv \frac{\|f\|}{\nu^{2} \lambda_{1}}
$$

In (4) $\|\cdot\|$ is the $\mathcal{L}^{2}$-norm and $\lambda_{1}=L^{-2}$ is the smallest eigenvalue of $-\Delta$ in the solution phase space, being the space of non-divergent zero-average vectorvalued functions on $\Omega$. These estimates rely on the asymptotic averages

$$
\begin{aligned}
& \left\langle\|u\|_{1}^{2}\right\rangle \equiv \limsup _{T \rightarrow \infty} \frac{1}{T} \int_{0}^{T} \mathrm{~d} t \int_{\Omega} u(-\Delta) u \mathrm{~d} x, \\
& \left\langle\|u\|_{2}^{2}\right\rangle \equiv \limsup _{T \rightarrow \infty} \frac{1}{T} \int_{0}^{T} \mathrm{~d} t \int_{\Omega}|\Delta u|^{2} \mathrm{~d} x .
\end{aligned}
$$

Note that $\nu\left\langle\|u\|_{1}^{2}\right\rangle$ and $\nu\left\langle\|u\|_{2}^{2}\right\rangle$ are, respectively, the average energy dissipation (hence, the average energy injection) and the average enstrophy ${ }^{4}$ dissipation

3 By definition, the Hausdorff dimension of a set is always bounded from above by its fractal dimension and the upper bound can in cases be strict. Nevertheless, the greatest lower bound on $m$ derived in this manner is an upper bound on both the fractal and Hausdorff dimensions of the global attractor (cf. [4,27]; [31], Appendix $\mathrm{B})$. Hence, there is no need to distinguish between the two dimensions in this context and the term "attractor dimension" hereafter may refer to either one.

4 The quadratic quantity $\|u\|_{1}^{2} / 2$ (or just $\|u\|_{1}^{2}$ ) is known in the classical theory of turbulence as the enstrophy. 
(hence, the average enstrophy injection). For fixed average forcing amplitude $\|f\| / L$ and fixed viscosity, $G \propto L^{3}$ so (2) and (3) are not linear in the domain area. Hence, these attractor-dimension estimates are not suggestive of extensive chaos although (3) is "essentially" extensive, only slightly "superextensive" by a logarithmic term.

However an intermediate step towards (2) is

$$
D \leq c_{1} \frac{\left\langle\|u\|_{1}^{2}\right\rangle^{1 / 2}}{\nu \lambda_{1}^{1 / 2}} .
$$

The energy equation gives the straightforward asymptotic inequality $\left\langle\|u\|_{1}^{2}\right\rangle^{1 / 2} \leq$ $\|f\| /\left(\nu \lambda_{1}^{1 / 2}\right)$, from which (7) then implies (2). As noted independently by Robinson [30] and Tran [34], the two attractor-dimension estimates (2) and (3) do not take into account the spatial scale of the forcing. A simple modification to the estimate of $\left\langle\|u\|_{1}^{2}\right\rangle$ improves (2) and gives it an explicit dependence on the forcing length scale (see $[2,30] ;[34]$, p.40). To this end let us consider the energy evolution equation

$$
\begin{aligned}
\frac{1}{2} \frac{\mathrm{d}}{\mathrm{d} t}\|u\|^{2} & =-\nu\|u\|_{1}^{2}+\int_{\Omega} u f \mathrm{~d} x=-\nu\|u\|_{1}^{2}+\int_{\Omega}(-\Delta)^{1 / 2} u(-\Delta)^{-1 / 2} f \mathrm{~d} x \\
& \leq-\nu\|u\|_{1}^{2}+\|u\|_{1}\|f\|_{-1} \leq-\frac{1}{2} \nu\|u\|_{1}^{2}+\frac{1}{2 \nu}\|f\|_{-1}^{2}
\end{aligned}
$$

where the two inequalities are obtained by applying the Schwarz and Young inequalities, respectively. It follows that asymptotically

$$
\left\langle\|u\|_{1}^{2}\right\rangle \leq \frac{1}{\nu^{2}}\|f\|_{-1}^{2}
$$

In terms of this estimate (7) becomes

$$
D \leq c_{1} \frac{\|f\|_{-1}}{\nu^{2} \lambda_{1}^{1 / 2}}
$$

This estimate, together with (3), which is written explicitly in terms of the physical parameters as

$$
D \leq c_{2}\left(\frac{\|f\|}{\nu^{2} \lambda_{1}}\right)^{2 / 3}\left(1+\ln \frac{\|f\|}{\nu^{2} \lambda_{1}}\right)^{1 / 3}
$$

are the focus of our present analysis. 
The norm $\|f\|_{-1}$ implicitly contains information about the spatial scale of $f$. For example, a monoscale forcing $f$, i.e. $-\Delta f=\lambda_{s} f$ where $\lambda_{s}$ is an eigenvalue of $-\Delta$, yields $\|f\|_{-1}=\lambda_{s}^{-1 / 2}\|f\|$. For a general forcing one may define the forcing length scale $\ell_{s} \equiv\|f\|_{-1} /\|f\|[34]$. Note that $\ell_{s}$ is always ${ }^{5}$ less than $L$, so that $\|f\|_{-1}<\|f\| / \lambda_{1}^{1 / 2}$. Hence, (10) is an improved estimate as compared to (2) (see [34], p.44). This fact is independently noted by Robinson [30].

We note in passing that while the energy injection decreases when a forcing with fixed $\|f\|$ is applied at progressively smaller length scales, the enstrophy injection $\int_{\Omega}(-\Delta) u f \mathrm{~d} x$ does not necessarily do so. Therefore, it does not seem possible to apply the above trick to improve (3), which is based on an estimate of the enstrophy injection.

Remark 1. The appearance of the forcing length scale in (10) is natural. The forcing scale has been shown to play a central role in the spectral distribution of energy and enstrophy and of their dissipation [36]. It also has non-trivial effects on optimal conditions for nonlinear stability [37].

Remark 2. It is a highly non-trivial problem to determine the energy injection rate $\int_{\Omega} u f \mathrm{~d} x$. This depends on how the fluid responds to a particular forcing $f$ and cannot be identified with $\|f\|^{2}$. The estimate of $\int_{\Omega} u f \mathrm{~d} x$ using the Schwarz and Young inequalities, leading to (9) and eventually to (10), provides what is essentially an upper bound on the energy injection rate. When a forcing $f$ with fixed $\|f\|$ is applied at progressively smaller scales, this upper bound decreases, leading to a decrease of the attractor-dimension estimate (10). The interpretation is that the decrease of the attractor dimension is due to the decrease of the energy injection, leading to an increase in the dissipation length scale (decrease of Reynolds number).

Remark 3. It is concluded in [30] that when a fixed amount of energy is injected at progressively smaller scales then the dimension of the attractor decreases. This conclusion seems to originate from the (mis)identification of $\|f\|^{2}$ with the energy injection rate. It is correct to say that a forcing with a fixed $\|f\|$ when applied at progressively smaller scales leads to a decrease of the attractor dimension, as seen above. However, such a forcing, when applied at progressively smaller scales, does not inject the same amount of energy and the interpretation in the preceding remark is more plausible.

Remark 4. To clarify the arguments in the previous two remarks let us consider the case of monoscale forcing $f$. This forcing gives rise to the (primary)

\footnotetext{
5 The case $\ell_{s}=L$ is pathological as the forcing then belongs to the eigenspace of $-\Delta$ corresponding to $\lambda_{1}$. This type of forcing is well known to lead to a trivial attractor consisting of a single stable stationary solution. This fact was first proven by Iudovich [17] and later investigated by Marchioro [25]. A simple proof is given by Constantin-Foias-Temam [7].
} 
stationary solution $\bar{u}=(-\nu \Delta)^{-1} f=f / \nu \lambda_{s}$ (see Remark 5), which is stable for sufficiently small $\|\bar{u}\|[37]$. In the stable regime, the energy injection rate is $\int_{\Omega} \bar{u} f \mathrm{~d} x=\|f\|^{2} /\left(\nu \lambda_{s}\right)=\|f\|_{-1}^{2} / \nu$. This energy injection rate indeed decreases with increasing $\lambda_{s}$ (decreasing forcing length scale), for a fixed $\|f\|$.

Remark 5. The fact that a monoscale forcing gives rise to a monoscale stationary solution is more readily deduced from the vorticity equation$\partial_{t} \Delta \psi+\partial_{x} \psi \partial_{y} \Delta \psi-\partial_{y} \psi \partial_{x} \Delta \psi=\nu \Delta^{2} \psi+g$, where $g=\widehat{z} \cdot \nabla \times f(\widehat{z}$ is the normal to the fluid domain) - than from (1). If $g$ satisfies $\Delta g=-\lambda_{s} g$, then $\bar{\psi}=-g /\left(\nu \lambda_{s}^{2}\right)$ is a stationary solution because the nonlinear terms identically vanish for $\psi=\bar{\psi}$. In the traditional NS system, $(u \cdot \nabla) u$ is non-zero (and not even non-divergent) for a non-divergent monoscale $u$. However, the divergent component of $(u \cdot \nabla) u$ is balanced by the pressure gradient term, and its non-divergent component vanishes for a non-divergent monoscale $u$. The latter component is described in the literature as the projection of the nonlinear term onto the non-divergent zero-average phase space, and is often denoted by $B(u, u)$. This projection is essentially equivalent to taking the curl of $(u \cdot \nabla) u$ to obtain the vorticity equation. Detailed treatments of $B(u, u)$ are given in $[23,37]$, where it is noted that the nonlinear interaction coefficient between two modes $k$ and $l$ contains the factor $\widehat{z} \cdot k \times l\left(|k|^{2}-|l|^{2}\right)[\widehat{z}$ is the normal to the wavevector plane]. (Pedlosky [28] notes that the nonlinear term in the vorticity equation vanishes for waves with the same wavelength or with parallel wavevectors.) Therefore, if the forcing $f$ in (1) consists of basic modes, for which the wavevectors are parallel or have the same magnitude, then $(-\nu \Delta)^{-1} f$ is a stationary solution. Thus, $\bar{u}$ is a stationary solution.

\section{Characteristic length scales}

There are various length scales that characterize the dynamics of a spatially extended system. Hohenberg and Shraiman [16] distinguish three length scales which are associated with excitation, dissipation, and correlation, and suggest that it is the ratios of these length scales to one another and to the typical system length scale that will determine the dynamics of the system. The excitation length scale is straightforward; it is the scale on which energy is injected into the system by external forcing. The dissipation length scale $\ell$ characterizes the length scale below which all modes are damped in a finite time. For fluid flow where molecular viscosity alone is responsible for dissipation, $\ell \sim(\nu \tau)^{1 / 2}$ where $\tau^{-1}$ is the local rate of shear [16]. This definition is applicable to both two- and three-dimensional flows. However, it is not the only definition of $\ell$ in each case. In two-dimensional flow, for example, Batchelor [3] and Kraichnan [20] define the dissipation length scale as $\ell=\left(\nu^{3} / \eta\right)^{1 / 6}$, where $\eta$ is the average enstrophy flux to small scales which asymptotically must equal the average rate of enstrophy dissipation $\nu\left\langle\|u\|_{2}^{2}\right\rangle / L^{2}$. It should be noted that these defini- 
tions have their roots entirely in physical and dimensional considerations. In particular, the latter definition is based on the hypothesis that the enstrophy cascades to small scales $(<\ell)$ via a $k^{-3}$ energy spectrum, so that $\ell$ is approximately identified with the length scale corresponding to the high-wavenumber end of the enstrophy-cascading range [20]. In the absence of a direct enstrophy cascade on the global attractor, as is argued in [36], this length scale loses its supposed physical relevance. The former definition, based on the local rate of shear, is associated with the energy dissipation instead of with the enstrophy dissipation, thereby not suffering from this difficulty. Nevertheless, given that there exists no dissipation length scale (for both energy and enstrophy) well separated from the forcing scale for a broad class of forcing [36], including the one considered by the classical theory of turbulence [20,22,29], the terminology "dissipation length scale" may not refer to where in wavenumber space the dissipation of energy (or enstrophy) occurs. Rather, it refers, in a qualitative sense, to the length scale below which viscous effects dominate nonlinearity. We will see later in this section that this length scale is better quantified by the concept of determining nodes $[13,14]$. In fact, it can be identified with a "minimum length scale" of the flow that can be rigorously deduced.

The concept of correlation length has a relatively long history in fluid dynamics (dating back to early in the 1900s), and in dynamical systems theory in general. There has been more than one definition of this length scale; two that are most relevant to the present discussion will be briefly reviewed. The first and simpler one is the two-point correlation length defined in terms of the correlation function

$$
C\left(x, x^{\prime}\right) \equiv\left\langle\left(\phi(x, t)-\langle\phi\rangle_{x}\right)\left(\phi\left(x^{\prime}, t\right)-\langle\phi\rangle_{x}\right)\right\rangle_{t},
$$

where the angle brackets with subscript $x$ denote the spatial average, the angle brackets with subscript $t$ denote the time average, and $\phi(x, t)$ is some local variable. In cases where $C\left(x, x^{\prime}\right) \sim \exp \left\{-\left|x-x^{\prime}\right| / \xi\right\}$ as $\left|x-x^{\prime}\right| \rightarrow \infty$ one can then define the two-point correlation length $\xi$. This length scale, if it exists, may be identified with the size of weakly-interacting subsystems. The second correlation length is called the dimension correlation length, the definition of which is due to Cross and Hohenberg [8]. According to the idea of extensive chaos, there exists a dimension density $\delta$ being the ratio of the attractor fractal dimension (or equivalently Hausdorff dimension) $D$ to the system volume $V$,

$$
\delta \equiv \frac{D}{V}
$$

Since $\delta$ has the physical units of inverse volume, Cross and Hohenberg [8] suggest defining a dimension correlation length $\xi_{\delta}$ : 


$$
\xi_{\delta} \equiv \delta^{-1 / d}
$$

where $d$ is the dimension of the system's physical space. It is highly likely that the dimension correlation length so defined might not be a new length scale. The justification for this claim lies in the heuristic argument in favor of extensive chaos provided in Section 1. It is argued there that the attractor dimension $D$ is of the order of $(L / \ell)^{d}$, so that $\delta=\ell^{-d}$. Substituting this into (12) one obtains $\xi_{\delta}=\ell$. Thus, def. (12) might just be the familiar dissipation length scale.

The present problem basically has four physical parameters, which can be independently controlled: the system linear length scale $L$, the forcing length scale $\ell_{s}$, the forcing root mean square amplitude (forcing density) $|f| \equiv\|f\| / L$, and the molecular viscosity $\nu$. The first two parameters make obvious and natural length scales. The last two parameters can be grouped, on dimensional grounds, to form a third length scale. Since the quantity $\left(\nu^{2} /|f|\right)^{1 / 3}$ has the physical units of length, it is tempting to designate this quantity as a dissipation length scale. It turns out, however, that by grouping $\nu,|f|$, and $\ell_{s}$ one can form a more physically plausible length scale which will be identified with the dissipation length scale $\ell$ :

$$
\ell=\left(\frac{\nu^{2}}{|f| \ell_{s}}\right)^{1 / 2}
$$

This agrees with the definition of $\ell$ in [16] because, according to (9), $|f| \ell_{s} / \nu$ is an estimate of (strictly, an upper bound for) the average rate of shear $\left\langle\|u\|_{1}^{2}\right\rangle^{1 / 2} / L$, thereby related to the energy dissipation (not enstrophy dissipation). Thus, this definition is expected to differ from the one due to Kraichnan and Batchelor. To see the extent of this difference, we write out the explicit expressions for these length scales, denoting the one due to Kraichnan and Batchelor by $\ell_{K B}$ and preserving the notation $\ell$ for the other,

$$
\begin{aligned}
\ell_{K B} & \approx\left(\frac{\nu L}{\left\langle\|u\|_{2}^{2}\right\rangle^{1 / 2}}\right)^{1 / 3} \\
\ell & \approx\left(\frac{\nu L}{\left\langle\|u\|_{1}^{2}\right\rangle^{1 / 2}}\right)^{1 / 2}
\end{aligned}
$$

It follows that

$$
\frac{\ell_{K B}^{3}}{\ell^{2}} \approx \frac{\left\langle\|u\|_{1}^{2}\right\rangle^{1 / 2}}{\left\langle\|u\|_{2}^{2}\right\rangle^{1 / 2}} .
$$


The right-hand side of (16) is the enstrophy dissipation length scale, which according to the classical theory $[3,20]$ would presumably be $\ell_{K B}$. This would imply $\ell_{K B} \approx \ell$. However, in the usual case of a spectrally localized forcing around a forcing length scale $\ell_{s}$, such that the energy and enstrophy injection rates are related by $\left\langle\int_{\Omega} u f \mathrm{~d} x\right\rangle=\ell_{s}^{2}\left\langle\int_{\Omega}(-\Delta) u f \mathrm{~d} x\right\rangle$, one has $\left\langle\|u\|_{1}^{2}\right\rangle^{1 / 2} /\left\langle\|u\|_{2}^{2}\right\rangle^{1 / 2}=\ell_{s}$ [36]. This result implies that $\ell_{K B} \approx\left(\ell^{2} \ell_{s}\right)^{1 / 3}$, which makes the difference between $\ell_{K B}$ and $\ell$ significant if $\ell \ll \ell_{s}$.

Closely related to the notion of dissipation length scale are the concepts of determining modes, nodes, and finite-volume elements $[11,13,14,18,19]$, from which a "minimum length scale" of the flow can be rigorously deduced. Here we restrict our discussion to the concept of determining nodes, which is introduced by Foias and Temam [13] and for which a sharp (sharpest to date) estimate of such a length scale is derived by Friz and Robinson [14]. A simplified version of Friz and Robinson's result can be stated as follows. Let $\left\{x_{i}\right\}_{i=1}^{n}$ be a set of $n$ lattice sites (nodes) equally spaced in the domain $\Omega$, so that the lattice spacing is $L / n^{1 / 2}$. Suppose that $u$ and $v$ are two trajectories on the global attractor and that $u$ and $v$ agree at the lattice sites, i.e. $u\left(x_{i}\right)=v\left(x_{i}\right)$, for $i=1,2, \cdots, n$. If this condition is satisfied, where $n$ is of the order of the attractor dimension $D$ and the forcing is analytic, then $u=v$. This result means that a trajectory on the global attractor is completely determined by its values at $D$ discrete nodes, hence the term "determining nodes". Obviously, the lattice spacing $L / D^{1 / 2}$ coincides with the dimension correlation length scale $\xi_{\delta}$ previously mentioned.

In terms of the length scales described in the preceding paragraphs, the results of the last section can be written as

$$
\begin{aligned}
& D \leq\left(\frac{L}{\ell}\right)^{2} \\
& D \leq\left(\frac{L^{3}}{\ell^{2} \ell_{s}}\right)^{2 / 3}\left(1+\ln \frac{L^{3}}{\ell^{2} \ell_{s}}\right)^{1 / 3}
\end{aligned}
$$

where a constant of order unity has been dropped from each expression. A couple of remarks are in order. First, not only does expression (17) agree with the idea of extensive chaos, but it also takes the form suggested by the heuristic argument in the introductory section. Second, it is clear that the logarithmic correction term in expression (18) surely diverges as $L \rightarrow \infty$; however, (18) may well be optimal for small domain size. In that case the system is slightly "super-extensive". When $L$ is sufficiently large, the bound on $D$ behaves extensively as (17) becomes optimal. In this regime, the attractordimension estimate grows linearly with the domain area, provided $\ell$ is fixed. Constancy of $\ell$ in a varying domain size requires fixed $|f|, \nu$, and $\ell_{s}$. A fixed $\ell_{s}$ means that the non-dimensional characteristic wavenumber of the forcing 
increases in exact proportion with $L$. Note that for the extensive regime (17) implies that $\ell \leq L / D^{1 / 2}$, so $\ell$ can be identified with the "minimum length scale" in the sense of Foias and Temam and of Friz and Robinson. For the super-extensive regime $\ell_{K B}$ plays the role of this length scale if the logarithmic correction is ignored. The correspondence between $\ell_{K B}$ and the rigorous "minimum length scale" (ignoring the logarithmic correction) has previously been noted by Friz and Robinson [14]. The length scale that separates the two regimes may be called the extensivity length scale [34]. Letting $L_{e}$ denote this length, a comparison of (17) and (18) gives

$$
\left(\frac{L_{e}}{\ell}\right)^{2} \sim\left(\frac{L_{e}^{3}}{\ell^{2} \ell_{s}}\right)^{2 / 3}\left(1+\ln \frac{L_{e}^{3}}{\ell^{2} \ell_{s}}\right)^{1 / 3}
$$

It follows that

$$
L_{e} \sim \exp \left\{\frac{\ell_{s}^{2}}{3 \ell^{2}}\right\}\left(\ell^{2} \ell_{s}\right)^{1 / 3},
$$

provided $\ell \ll \ell_{s}$. It can be confirmed that $L_{e}>\ell_{s}$, so a non-trivial extensivity length scale does exist.

Finally, the dimension correlation length as defined by Cross and Hohenberg [8] and advanced by Egolf $[9,10]$ is just the newly defined dissipation length $\ell$, which has the physical significance as discussed above. In particular, $\ell$ represents the spacing of determining nodes, and in the absence of a dissipation range well separated from the forcing region [36], the terminology associated with $\ell$ finds itself a better justification in this new sense.

\section{Conclusion}

We have analysed the two standard attractor-dimension estimates of the twodimensional Navier-Stokes equations, derived by Constantin-Foias-Temam. It is shown that one of the estimates grows linearly with the domain area, for sufficiently large systems, when the kinematic viscosity and forcing density and its length scale are held fixed. This is consistent with the central idea of extensive chaos: namely the linear scaling of complexity, as characterized by the attractor dimension, with domain area. This qualifies (10) as the optimal estimate for large systems. For small systems, for which the domain length scale is below a threshold called the extensivity length scale, the estimate (11) becomes optimal. In this regime, the attractor dimension is slightly superextensive, essentially by a logarithmic factor. 
In the extensive regime, the upper bound on the attractor dimension is given by the ratio of the domain area to the square of the dissipation length scale, which is defined, on physical grounds, in terms of the average shear. This implies an equivalence between the dissipation length scale and the dimension correlation length scale. The latter has been suggested to be a new characteristic length scale for extensively chaotic systems, but it seems likely that it is just another way of determining the dissipation length scale.

\section{Acknowledgements}

The work reported here represents part of CVT's Ph.D. thesis at the University of Toronto, which was supported by University of Toronto Open Fellowships and Department of Physics Burton Fellowships. TGS would like to acknowledge support from the Natural Sciences and Engineering Research Council and the Meteorological Service of Canada. Constructive comments from a reviewer were very much appreciated.

\section{References}

[1] I.S. Aranson, A.V. Gaponov-Grekhov, M.I. Rabinovich, The development of chaos in dynamic structure ensembles, Sov. Phys. JETP 62 (1985) 52-59.

[2] A.V. Babin, M.I. Vishik, Attractors of partial differential equations and estimate of their dimensions, Russian Math. Surveys 38:4 (1983) 151-213.

[3] G.K. Batchelor, Computation of the energy spectrum in homogeneous twodimensional turbulence, Phys. Fluids 12 (II) (1969) 233-239.

[4] V.V. Chepyzhov, A.A. Ilyin, A note on the fractal dimension of attractors of dissipative dynamical systems, Nonlinear Anal. 44 (2001) Ser. A: Theory Methods, 811-819.

[5] P. Constantin, C. Foias, Navier-Stokes Equations, University of Chicago Press, Chicago, 1988.

[6] P. Constantin, C. Foias, R. Temam, Attractors representing turbulent flows, Mem. Am. Math. Soc. 53 (314) (1985) 1-67.

[7] P. Constantin, C. Foias, R. Temam, On the dimension of the attractors in twodimensional turbulence, Physica D 30 (1988) 284-296.

[8] M.C. Cross, P.C. Hohenberg, Pattern formation outside of equilibrium, Rev. Mod. Phys. 65 (1993) 851-1112.

[9] D.A. Egolf, Characterization of extensively chaotic states and transitions, Ph.D. thesis, Department of Physics, Duke University, Durham, NC, 1994.

[10] D.A. Egolf and H.S. Greenside, Relation between fractal dimension and spatial correlation length for extensive chaos, Nature 369 (1994) 129-131. 
[11] C. Foias, O.P. Manley, R. Temam, Y.M. Treve, Asymptotic analysis of the Navier-Stokes equations, Physica D 9 (1983) 157-188.

[12] C. Foias, R. Temam, Some analytic and geometric properties of the solutions of the Navier-Stokes equations, J. Math. Pure Appl. 58 (1979) 339-369.

[13] C. Foias, R. Temam, Determination of the solutions of the Navier-Stokes equations by a set of nodal values, Math. Comp. 43 (1984) 117-133.

[14] P.K. Friz, J.C. Robinson, Parametrising the attractor of the two-dimensional Navier-Stokes equations with a finite set of nodal values, Physica D 148 (2001) 201-220.

[15] H.S. Greenside, Spatiotemporal chaos in large systems: the scaling of complexity with size. Preprints of the Montreal workshop of the CRM (Centre de Recherche en Mathematiques) under the title "Semi-analytic methods for the NavierStokes equations", Oct. 1995.

[16] P.C. Hohenberg, B.I. Shraiman, Chaotic behaviour of an extended system, Physica D 37 (1989) 109-115.

[17] V.I. Iudovich, Example of the generation of a secondary stationary or periodic flow when there is loss of stability of the laminar flow of a viscous incompressible fluid, J. Appl. Math. Mech. 29 (1965) 527-544.

[18] D.A. Jones, E.S. Titi, Determining finite volume elements for the 2D NavierStokes equations, Physica D 60 (1992) 165-174.

[19] D.A. Jones, E.S. Titi, Upper bounds on the number of determining modes, nodes, and volume elements for the Navier-Stokes equations, Indiana Univ. Math. J. 42 (1993) 875-887.

[20] R.H. Kraichnan, Inertial ranges in two-dimensional turbulence, Phys. Fluids 10 (1967) 1417-1423.

[21] O.A. Ladyzhenskaya, The Mathematical Theory of Viscous Incompressible Flow, Gordon and Breach, New York, 2nd ed., 1969.

[22] M. Lesieur, Turbulence in Fluids, 3rd edition, Kluwer, Dordrecht, 1997.

[23] V.X.S. Liu, A sharp lower bound for the Hausdorff dimension of the global attractors of the 2D Navier-Stokes equations, Comm. Math. Phys. 158 (1993) $327-339$.

[24] P. Manneville, Lyapunov exponents for the Kuramoto-Sivashinsky model, Macroscopic modeling of turbulent flows (O. Pironneau, ed.), Lecture Notes in Physics, vol. 230, Springer-Verlag, New York, 1985, 319-326.

[25] C. Marchioro, An example of absence of turbulence for any Reynolds number, Comm. Math. Phys. 105 (1986) 99-106.

[26] C. O'Hern, D. Egolf, H. Greenside, Lyapunov spectral analysis of a nonequilibrium Ising-like transition, Phys. Rev. E 53 (1996) 3374-3386. 
[27] B.R. Hunt, Maximum local Lyapunov dimension bounds the box dimension of chaotic attractors, Nonlinearity 9 (1996) 845-852.

[28] J. Pedlosky, Geophysical Fluid Dynamics, 2nd edition, Springer-Verlag, New York, 1987.

[29] A. Pouquet, M. Lesieur, J.C. André, and C. Basdevant, Evolution of high Reynolds number two-dimensional turbulence, J. Fluid Mech. 72 (1975) 305319 .

[30] J.C. Robinson, Low dimensional attractors arise from forcing at small scales, Physica D 181 (2003) 39-44.

[31] J.C. Robinson, Infinite-Dimensional Dynamical Systems, Cambridge University Press, New York, 2001.

[32] R. Temam, Infinite-Dimensional Dynamical Systems in Mechanics and Physics, Springer-Verlag, New York, 2nd ed., 1997.

[33] R. Temam, Navier-Stokes Equations and Nonlinear Functional Analysis, SIAM, Philadelphia, 1983.

[34] C.V. Tran, Extensive Chaos and Complexity of Two-Dimensional Turbulence, Ph.D. Thesis, University of Toronto, 2001.

[35] C.V. Tran, J.C. Bowman, On the dual cascade in two-dimensional turbulence, Physica D 176 (2003) 242-255.

[36] C.V. Tran, T.G. Shepherd, Constraints on the spectral distribution of energy and enstrophy dissipation in forced two-dimensional turbulence, Physica D 165 (2002) 199-212.

[37] C.V. Tran, T.G. Shepherd, H.-R. Cho, Stability of stationary solutions of the forced Navier-Stokes equations on the two-torus, Discrete Contin. Dyn. Syst. Ser. B 2 (2002) 483-494.

[38] M. Ziane, Optimal bounds on the dimension of the attractor of the NavierStokes equations, Physica D 105 (1997) 1-19. 